\title{
Grundzüge einer quartären Bodenstratigraphie in Süddeutschland
}

\author{
Von K. Brunnacker, Köln \\ Mit 1 Tabelle im Text
}

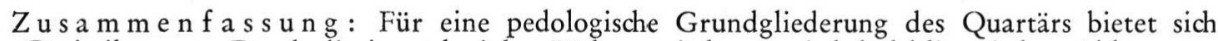
eine Dreiteilung an. Durch die interglazialen Böden zwischen typisch kaltklimatischen Ablagerungen glazigener, fluvioglazialer und vor allem periglazialer Fazies wird das Quartär vom Günz ab bis zum Postglazial stratigraphisch erfaßt. Davor liegt ein Abschnitt, der pedologisch durch Riesenböden und geologisch wie geomorphologisch z. T. durch mächtige Talverschüttungen gekennzeichnet ist. Ein ältester Quartärabschnitt zeichnet sich durch Reliktböden aus. Während noch beim mittleren Abschnitt einige Aussagen über die kühleren Zwischenphasen gemacht werden können, ist es beim ältesten Abschnitt bislang nicht möglich gewesen, die zugehörigen Kaltzeiten (i.e. S.) direkt zu erfassen.
\end{abstract}

S u m mary : Quaternary deposits of southern Germany can roughly be divided into 3 units. From Günz to Postglacial time interglacial soil formations occur inbetween deposits of typical low-temperature environment (such as of a morainal, fluvioglacial or most of all periglacial facies). Thus various Quaternary formations can be established.

The Pregünz is characterised by a unit, which appears to be significant because of its particularily deeply weathered soils (= Riesenböden). The same time is marked by geological and geomorphological changes which led for excample to considerable accumulations in former valleys. The oldest soil formation of Quaternary age which could be noticed is marked by relicts of deep soil formation (= Reliktböden).

Faunen- und Florenreste, manchmal auch Zeugnisse menschlicher Betätigung, sind innerhalb quartärer Ablagerungen willkommene Alterskriterien. Leider treten sie nur höchst vereinzelt und oft ohne eindeutige stratigraphische Aussage auf. Sie vermögen aus diesem Grunde insbesondere im nördlichen Alpenvorland auf die Quartärgliederung keinen nachhaltigen Einfluß auszuüben. Im einstigen Vergletscherungsgebiet der Alpen und in dessen Vorland baute die Stratigraphie deshalb von Anfang an auf den Moränen und den zugehörigen Schottern auf. Diese Bildungen, denen kaltklimatische Genese unterstellt wird, bei den Schottern aber durchaus nicht immer erwiesen ist, werden dabei direkt erfaßt. Die dazwischenliegenden Warmzeiten werden, von einigen Ausnahmen abgesehen, nur indirekt einbezogen - insbesondere über zwischengeschaltete Erosionsphasen.

Unter Berücksichtigung der bekannten pedologischen Kriterien kann zumindest in der Theorie auch umgekehrt vorgegangen werden, indem man die durchaus nicht raren interglazialen Böden zur Basis einer Quartärgliederung wählt. Ein derartiges in Süddeutschland entwickeltes bodenstratigraphisches Arbeitsschema soll im folgenden kurz dargestellt werden - kurz deshalb, weil es sich um die Zusammenfassung mehrerer Spezialuntersuchungen handelt und weil die klimatische Bewertung dieser Böden wie die Größenordnung ihrer Zeitdauer an anderer Stelle bereits erörtert wurde (vgl. K. BrunNaCKEr 1964c). Zudem dürften sich im Laufe der Zeit noch Ergänzungen und kleinere Änderungen ergeben (s. Tab. 1).

\section{Das jüngere Pleistozän im nördlichen Alpenvorland}

Die im nördlichen Alpenvorland in den Interglazialen Günz/Mindel, Mindel/Riß und Riß/Würm vorkommenden Böden sind weitaus überwiegend als Parabraunerden, bei entsprechendem Substrat auch mit Übergängen zur Terra fusca entwickelt. Bei der Decklehmfazies des Lösses kommt der Pseudogley hinzu. Außerdem sind die aus Löß entstandenen Parabraunerden mehr oder weniger stark pseudovergleyt. Die Ausbildung dieser Böden 
Tabelle 1

Bodenstratigraphische Gliederung des Quartärs in Süddeutschland

\begin{tabular}{|c|c|c|c|}
\hline & Deckschichten & Talverschüttungen & $\begin{array}{l}\text { Verebnungsflächen } \\
\text { (z. B. Sandstein- } \\
\text { keupergebiet in } \\
\text { Mittelfranken) }\end{array}$ \\
\hline $\begin{array}{l}\text { Postglazial } \\
\text { Würm }\end{array}$ & $\begin{array}{l}\text { Parabraunerde } \\
\text { Löß }\end{array}$ & \multirow{4}{*}{$\begin{array}{l}\text { Deckschichten in } \\
\text { entsprechender } \\
\text { Ausbildung }\end{array}$} & \multirow{7}{*}{$\begin{aligned}\left\{\begin{array}{l}\text { Anschluß nicht } \\
\text { voll gesichert }\end{array}\right. \\
\\
\text { "Auenboden“, } \\
\text { Gley mit ausgepräg- } \\
\text { tem Go- bzw. Gr- } \\
\text { Horizont }\end{aligned}$} \\
\hline $\begin{array}{l}\text { R/W-I. } \\
\text { Riß }\end{array}$ & $\begin{array}{l}\text { Parabraunerde } \\
\text { Löß und Lößlehm }\end{array}$ & & \\
\hline $\begin{array}{l}\text { M/R-I. } \\
\quad \text { Mindel }\end{array}$ & $\begin{array}{l}\text { Parabraunerde } \\
\text { Lößlehm u. Deck- } \\
\text { lehm }\end{array}$ & & \\
\hline $\begin{array}{l}\text { G/M-I. } \\
\text { Günz }\end{array}$ & $\begin{array}{l}\text { Parabraunerde } \\
\text { Lößlehm u. Deck- } \\
\text { lehm }\end{array}$ & & \\
\hline $\begin{array}{l}\text { Zeit der „Riesenbö- } \\
\text { den“ und der Talver- } \\
\text { schüttung (entspre- } \\
\text { chend Mauer, Mos- } \\
\text { bach usw.) }\end{array}$ & & $\begin{array}{l}\text { Pseudogley } \\
\text { Lokalsedimente } \\
\text { extremer Pseudogley } \\
\text { Talsedimente } \\
\text { mächtiger Auenboden } \\
\text { Talsedimente } \\
\text { Erosionsphase }\end{array}$ & \\
\hline $\begin{array}{l}\text { Zeit der Reliktböden; } \\
\text { Donaukaltzeiten dar- } \\
\text { in vorläufig nicht ge- } \\
\text { nau einstufbar }\end{array}$ & & \multirow{2}{*}{$\begin{array}{l}\text { Anschluß nicht } \\
\text { erfaßt }\end{array}$} & \\
\hline Pliozän & & & \\
\hline
\end{tabular}

ist in diesem Gebiet im Prinzip zu gleichartig, als daß sich Leithorizonte innerhalb der genannten Warmzeiten für ein bestimmtes Interglazial abzuzeichnen vermöchten, zumal die Böden meist erodiert sind und deshalb auch kaum über die primäre Entwicklungstiefe Aufschluß zu geben vermögen.

Die Bodenstratigraphie fußt hier auf zwei Möglichkeiten, einmal auf der Auswertung der periglazialen Deckschichten und der darin eingeschalteten Böden, zum andern auf der Identifikation von Bodenresten, die zwischen Moränen und Schottern liegen.

Im Idealfall ist jede der drei interglazialen Warmzeiten, die einer eiszeitlichen Moränen- und Schotterakkumulation nachfolgen, durch einen entsprechenden Boden und jede Eiszeit durch eine periglaziale Deckschicht vertreten. Bei der Auswertung dieser Deckschichten ist allerdings zu berücksichtigen, daß sich damit nur das Mindestalter der Basisschicht erfassen läßt. Dies gilt vor allem für das Altmoränengebiet, wo wenigstens zeitweise der periglaziale Abtrag dominant war. Es gilt aber auch für weite Schotterfluren, die zwischendurch ohne Löß- und Fließerdeeindeckung geblieben sind. Nur in günstigsten Fällen kommt man deshalb auf diesem Wege über die drittletzte Eiszeit noch etwas zurück.

Im Moränengebiet, wo die glazigenen Bildungen z. T. nicht wie in der Terrassenlandschaft ineinandergeschachtelt sind, sondern übereinandergestapelt liegen, treten manchmal 
fossile Bodenreste zwischen den Schottern und Moränen auf. Die Abzählmethode ist hier nicht am Platze; denn es vermögen zusätzliche, nicht ohne weiteres erkennbare Lücken auf treten.

In jedem Falle muß also der hangende Schotter (oder Moräne) eine eigene stratigraphische Sicherung erfahren, sei es geologisch oder geomorphologisch oder über die genannte Deckschichtenanalyse. Aber auch das Liegende des fossilen Bodens muß nicht unbedingt die unmittelbar vorangegangene Eiszeit repräsentieren. Der Boden kann sich auch über mehrere Interglazialzeiten hinweg gebildet haben, wenn eine periglaziale Eindeckung zwischendurch unterblieben ist. Die Anwendung dieser zweiten Methode hat vor allem in Oberschwaben gute Ergebnisse gebracht (H. Graul 1962).

\section{Die Zeit der großen Talverschüttung in Süddeutschland}

Die Riesenböden, die im Zusammenhang mit der großen Talverschüttung in Süddeutschland vorkommen, entsprechen in ihrer typologischen Ausbildung derjenigen, welche für die interglazialen Böden des jüngeren Quartärs kennzeichnend ist - nämlich der Terra fusca, dem Pseudogley, den Auenböden und einem braunerdeartigen Boden. Sie weichen jedoch hinsichtlich ihrer Mächtigkeit und ihres Ausprägungsgrades von den jüngeren Interglazialböden so erheblich $a b$, daß man berechtigt ist, von $R$ i e s e $n$ b ö d en zu sprechen. Die Ursache dieser extremen Ausbildung kann nicht in einer örtlich sehr gesteigerten Intensität der Bodenbildung, sondern allein in einer entsprechend langen Dauer der Bodenbildungszeit gesucht werden, da diese Böden stratigraphisch einem bestimmten Zeitabschnitt zugeordnet werden müssen (vgl. K. BrunNaCKer 1962, 1964 a u. b, H. Graul 1962). Uber die Deckschichtenfolge lassen sich zwei derartige Böden bei Regensburg vor die Günzeiszeit einstufen. Das gleiche Ergebnis zeigt sich in Oberschwaben mittels der zweiten genannten Methode (H. Graul 1962).

\section{Die Reliktflächen}

Vor allem im Gebiet des Sandsteinkeupers von Mittelfranken und Ostwürttemberg, aber auch im Bereich der Gipfelflur des Tertiärhügellandes bei Freising und vermutlich auch auf mesozoischen Sandsteinen des Naabschollenlandes treten Reliktböden auf (S. Müller 1958, K. Brunnacker 1961, 1962), die ursprünglich von mir als endtertiäre Bildungen angesehen wurden. Die weitergehenden, auch heute noch nicht abgeschlossenen Untersuchungen in Mittelfranken haben aber gezeigt, daß es sich um ältestquartäre Bildungen handeln muß (vgl. K. BRunNacker 1964b). Die Schwierigkeit ihrer Datierung besteht darin, daß sie einerseits nur in Flußferne erhalten sind, andererseits aber auf quartäre Terrassensysteme bezogen werden müssen - entsprechenden Problemen steht auch J. Fink (1960/1961) am Ostrand der Alpen gegenüber. In Mittelfranken ist z. B. am ehesten über das Rednitztal eine Verknüpfung mit den Ablagerungen des Maintales möglich. Ein derartiges Vorgehen erfordert aber die Neubearbeitung der Rednitzterrassen. Insgesamt zeichnet sich trotz dieser Schwierigkeiten eine Einstufung der Reliktböden in die Zeit vor der großen Talverschüttung ab (Tab. 1). Diese Reliktböden sind zumeist ähnlich den rezenten Auenböden und dem Gley ausgebildet. Allerdings wird für den erstgenannten Boden eine abweichende, klimabedingte Genese unterstellt (vgl. K. BrunNacker 1964 c).

Das Hauptproblem der Bodenstratigraphie besteht somit darin, die Reliktböden einmal mit den Böden des nachfolgenden Quartärs besser als bisher zu verknüpfen und zum andern einen pedologischen Anschluß an das jüngste Pliozän zu finden.

\section{Die Bodenreste des jüngsten Pliozäns}

Außerst spärlich und genetisch problematisch sind bis jetzt die terrestrischen Böden zu Beurteilen, die dem jüngsten Pliozän zugehören. Der aus dem Untermaintal durch 
H. KöRBER (1962) erwähnte Bodenrest verspricht mit am ehesten eine Aussage; er wird derzeit neu bearbeitet. Ob hingegen die von K. D. Duphorn (1961) behandelte Roterde des östlichen Rhönvorlandes eine echte paläoklimatische Aussage, auf die es vorläufig allein ankommt, gestattet, scheint noch ungewiß. Es handelt sich um einen Boden aus Aschetuffit, und dabei ist $\mathrm{zu}$ bedenken, daß basaltisches und verwandtes Material eine gewisse Vorzugstendenz zur Bildung roter Böden zu geben scheint.

\section{Vergleich mit anderen Gebieten}

Für den großräumigen Vergleich einer Bodenstratigraphie des Pleistozäns können die eingangs genannten drei Hauptabschnitte zugrundegelegt werden. Der heutige Kenntnisstand reicht nämlich nur zum Teil aus, hinsichtlich der Einzelheiten zu weiträumigen Detailvorstellungen zu kommen. Am ehesten finden sich Parallelen mit den Ergebnissen von J. FINK (1960) im außer- und randalpinen Bereich O'sterreichs. Hier treten ebenfalls oberpliozäne Rotlehme aus Basalt auf. Im Burgenland sind als ältestquartäre Bildungen ebenfalls vergleichbare Reliktböden vorhanden. Böden, die sich mit unseren Rinsenböden des mittleren Abschnittes vergleichen lassen, scheinen über der Wienerbergterrasse und eventuell zwischen deren Deckschichten zu liegen (vgl. H. KüPPER 1955). Die jüngeren Interglazialböden lassen sich im allgemeinen ebenfalls vergleichen. Eine Sondersteilung nimmt allein die pedologische Ausprägung der Göttweiger Bodenbildung des Riß/Würm-Interglazials in Niederösterreich und im angrenzenden Mähren ein. Doch läßt sich dies mit paläoklimatischen Erwägungen durchaus erklären (vgl. K. BrunNaCKer 1964).

Eine andere Bodenstratigraphie, die auf den sog. Terrae calcis aus Kalkgestein fußt. wurde in den letzten Jahren in den Karpathen durch L. SMoliková \& V. LožEK (1962) begründet. Danach findet sich im Oberpliozän eine allitische Terra rossa, hingegen eine siallitische T erra rossa im ältesten Quartär, danach eine tiefgründige Terra fusca und schließlich in den jüngeren Interglazialen eine geringmächtige Terra fusca. Zumindest im Prinzip ist also auch hier eine entsprechende Aufgliederung möglich. Ein direkter Vergleich mit unserem Gebiet scheitert daran, daß hier die Terra rossa-Bildung im wesentlichen schon im Obermiozän zu Ende gegangen ist (vgl. F. Birzer 1939, K. BrunNaCKen 1959). Außerdem liegt für das Quartär, wo noch in den älteren Abschnitten eine echte Terra fusca aus Kalkstein entstehen konnte (bis in den mittleren Abschnitt hinein), verhältnismäßig wenig Beobachtungsmaterial vor. Gemäß den Ausführungen von M. KRETzOI (1961) scheint aber andererseits nach Südosteuropa hin die Bildungstendenz der Terra rossa noch etwas weiter in das Quartär hinein anzuhalten. In Südwesteuropa konnte H. Alimen (1954) über die Intensität der Bodenfarben ähnliche Feststellungen machen, die darauf hinauslaufen, daß mit Annäherung an die Gegenwart die Verwitterungsintensität zunehmend abgeschwächt wurde.

Wenngleich die Bodenstratigraphie des Quartärs noch in den Anfängen steht, so vermag sie doch heute schon manche Gesetzmäßigkeit aufzuzeigen. Um aber echte Fortschritte bringen zu können, muß eine solche Bodenstratigraphie auch die Ergebnisse der bisherigen über andere Methoden gewonnenen Erkenntnisse einbeziehen. Das mindeste, was sich bei einem solchen Vorgehen zeigt, sind eventuell vorhandene Lücken in der stratigraphischen Interpretation.

\section{Literaturverzeichnis}

Alimen, H.: Colorimétrie de sédiments quaternaires et paleoclimats - Premier resultats. - Bull. Soc. geol. France 6, 1954.

BirzeB, F.: Verwitterung und Landschaftsentwicklung in der südlichen Frankenalb. - Z. deutsch. geol. Ges. 91, S. 1-57, Berlin 1939. 
BRUNNACKER, K.: Jungtertiäre Böden in Nordbayern. - Geol. Bl. NO-Bayern 9, S. 55-63, Erlangen 1959. - - Reliktböden im östlichen Mittelfranken. - Geol. Bl. NO-Bayern 12, S. 183-190, Erlangen 1962 [1962a]. - - Pleistozäne Böden im nördlichen Oberschwaben. - In GraUL, H.: Eine Revision der pleistozänen Stratigraphie des schwäbischen Alpenvorlandes (S. 255-259). - Peterm. geogr. Mitt., S. 253-271, Gotha 1962 [1962b]. - - Böden des älteren Pleistozäns bei Regensburg. - Geologica Bavarica 53, S. 148-160, München 1964 [1964a]. - - Über Ablauf und Altersstellung altquartärer Verschüttungen im Maintal und nächst dem Donautal bei Regensburg. - Eiszeitalter und Gegenwart 15, Öhringen 1964 [1964b]. - - Schätzungen über die Dauer des Quartärs, insbesondere auf der Grundlage seiner Paläoböden. - Geol. Rdsch. 1964 (im Druck) [1964c].

DuphoRN, K. D.: Sedimentologische Untersuchung der plio-pleistozänen Grenzschichten im östlichen Vorland der Hohen Rhön. - Diss. Univ. Würzburg 1961.

FINK, J.: Leitlinien einer österreichischen Quartärstratigraphie. - Mitt. geol. Ges. Wien 53, S. 249266, Wien 1960. - - Die Südostabdachung der Alpen - Exkursionen durch Ósterreich -. Mitt. österr. bodenkundl. Ges. 6, S. 123-183, Wien 1961.

Graul, H.: Eine Revision der pleistozänen Stratigraphie des schwäbischen Alpenvorlandes. Peterm. geogr. Mitt., S. 253-271, Gotha 1962.

KöRBER, H.: Die Entwicklung des Maintals. - Würzburger geograph. Arb. 10, Würzburg 1962.

KretzoI, M.: Stratigraphie und Chronologie - Stand der Ungarischen Quartärforschung. - Inst. Geolog. Prace 34, S. 313-330, Warszawa 1961.

KüPPER, H.: Ausblick auf das Pleistozän des Raumes von Wien - Beiträge zur Pleistozänforschung in Österreich. - Verh. geol. Bundesanst., Sonderh. D, S. 136-152, Wien 1955.

MülLER, S.: Feuersteinlehme und Streuschuttdecken in Ostwürttemberg. - Jh. geol. L.-A. BadenWürttemberg 3, S. 241-262, Freiburg i. Br. 1958.

Smolikoví, L. \& LožEk, V.: Zur Altersfrage der mitteleuropäischen Terrae calcis. - Eiszeitalter und Gegenwart 13, S. 157-177, O'hringen 1962.

Manuskr. eingeg. 1. 12. 1964

Anschrift des Verf.: Prof. Dr. K. Brunnacker, Geolog. Institut der Univ., 5 Köln, Zülpicherstr. 49. 